Institute for Computational Mathematics

Hong Kong Baptist University

ICM Research Report

11-03 


\title{
High Order Weighted Essentially Non-Oscillation Schemes for Two-Dimensional Detonation Wave Simulations
}

\author{
Zhen Gao $\stackrel{*}{,}$ Wai Sun Don † Zhiqiu Li ${ }^{\ddagger}$
}

August 1, 2011

\begin{abstract}
In this paper, we demonstrate the detailed numerical studies of three classical two dimensional detonation waves by solving the two dimensional reactive Euler equations with species with the fifth order WENO-Z finite difference scheme (JCP 227, 2008) with various grid resolutions. To reduce the computational cost and to avoid wave reflection from the artificial computational boundary of a truncated physical domain, we derive an efficient and easily implemented one dimensional Perfectly Matched Layer (PML) absorbing boundary condition (ABC) for the two dimensional unsteady reactive Euler equation when one of the directions of domain is periodical and inflow/outflow in the other direction. The numerical comparison among characteristic, free stream, extrapolation and PML boundary conditions are conducted for the detonation wave simulations. The accuracy and efficiency of four mentioned boundary conditions are verified against the reference solutions which are obtained from using a large computational domain. Numerical schemes for solving the system of hyperbolic conversation laws with a single-mode sinusoidal perturbed ZND analytical solution as initial conditions are presented. Regular rectangular combustion cell, pockets of unburned gas and bubbles and spikes are generated and resolved in the simulations. It is shown that large amplitude of perturbation wave generates more fine scale structures within the detonation waves and the number of cell structures depends on the wave number of sinusoidal perturbation.
\end{abstract}

\section{Keywords}

Weighted Essentially Non-Oscillatory, Detonation, ZND, Perfectly Matched Layer

\section{AMS}

$65 \mathrm{P} 30,77 \mathrm{Axx}$

\footnotetext{
${ }^{*}$ Correspondence author: School of Mathematical Sciences, Ocean University of China, Qingdao, China. E-Mail: zhengao@ouc.edu.cn

${ }^{\dagger}$ Department of Mathematics, Hong Kong Baptist University, Hong Kong, China. E-Mail: wsdon@hkbu.edu.hk

${ }^{\ddagger}$ Department of Mathematics, Hong Kong Baptist University, Hong Kong, China. E-Mail: zhiqiuli@hkbu.edu.hk
} 


\section{Introduction}

Conservative Weighted Essentially Non-Oscillation finite difference schemes (WENO) have been developed in recent years as a class of high order/high resolution method for solutions of hyperbolic conservation laws (PDEs) in the presence of shocks and small scale structures in the solution (for details and history of WENO scheme, see [1] and references contained therein). WENO schemes owe their success to the use of a dynamic set of substencils where a nonlinear convex combination of lower order polynomials adapts either to a higher order polynomial approximation at smooth parts of the solution, or to a lower order polynomial approximation that avoids interpolation across discontinuities. The upwinding of the spatial discretization provides the necessary dissipation for shock capturing. It is an evolution of the Essentially Non-Oscillatory (ENO) schemes, introduced in [2], which choose only the smoothest substencil, instead of forming a convex combination of all the substencils available in order to optimize accuracy in the smooth parts of the solution.

The local computational stencils of $(2 r-1)$ order WENO schemes are composed of $r$ overlapping substencils of $r$ points, forming a larger stencil with $(2 r-1)$ points. The scheme yields a local rate of convergence that goes from order $r$ at the non-smooth parts of the solution, to order $(2 r-1)$ when the convex combination of local lower order polynomials is applied at smooth parts of the solution. The nonlinear coefficients of WENO's convex combination, hereafter referred to as nonlinear weights $\omega_{k}$, are based on lower order local smoothness indicators $\beta_{k}, k=0, \ldots, r-1$ that measure the sum of the normalized squares of the scaled $L^{2}$ norms of all derivatives of $r$ local interpolating polynomials. An essentially zero weight is assigned to those lower order polynomials whose underlining substencils contain high gradients and/or shocks, aiming at an essentially nonoscillatory solution close to discontinuities. At smooth parts of the solution, the formal order of accuracy is achieved through the mimicking of the central upwind scheme of maximum order, when all smoothness indicators are about the same size. Hence, the one of the most important issues for WENO schemes is to design an efficient and accurate nonlinear weights $\omega_{k}$. In [3], the first set of nonlinear weights of widespread use has been given. However, it has been shown that the nonlinear weights fail to satisfy the necessary and sufficient condition for achieving the formal order of accuracy even for smooth functions. We call this scheme as the classical WENO scheme (WENO-JS). In [4], a modification of the nonlinear weights was proposed in the form of a mapping on the classical WENO-JS nonlinear weights, leading to corrected nonlinear weights that recovered the formal order of accuracy. We call the scheme composed by this mapped set of nonlinear weights as the mapped WENO scheme (WENO-M). In [5], it was shown that the incorporation of a global optimal order smoothness indicator, hereafter denoted as $\tau_{2 r-1}$, into the classical WENO-JS nonlinear weights definition satisfies the necessary and sufficient condition for achieving the formal order of accuracy. This scheme has been named the WENO-Z scheme (WENO-Z). The mapping procedure of WENO-M incurs extra expensive computational cost, while the weight modification of WENO-Z is obtained through a simple and inexpensive linear combination of the already computed lower order local smoothness indicators $\beta_{k}$. It has been shown that the new set of nonlinear weights of WENO-Z provided less dissipation than WENO-JS and yielded comparable resolution of smooth solution and captured sharp gradients as WENO-M [5]. 
Since the classical theory, namely the ZND detonation model, in detonation waves was pioneered by Zekdivich [6], von Neumann [7] and Doering [8], the studies of detonation waves have been an active area of research in both theoretical studies and in numerical simulations due to the practical importance. For instance, linear stability analysis [9] presented rigorous results, but it is unable to capture properties of non-linear dynamics. In the 1970s, experiments $[10,11,12]$ demonstrated that the detonations observed in many circumstances exhibited complicated unstable wave patterns in reacting gases. Numerical approaches such as the second order Godunov scheme[13, 14], extended CE/SE method [15], unsplit scheme [16], classical WENO-JS scheme [17, 18] have been implemented to simulate two dimensional detonation waves to investigate the detonation phenomenon in many physical applications. Moreover, the grid convergence study for the case of overdrive factor $f=1.6$ in [19] showed that the WENO schemes converge faster than existing numerical methods such as PPM with front tracking and mesh refinement [20], unsplit scheme [21], Roe's solver with minmod limiter [22] and Roe's solver with superbee limiter [22]. Consequently, in this paper we are interested in employing the fifth order WENO-Z schemes for the numerical simulations of two dimensional detonation waves.

In simulating detonation waves in a semi-infinite physical domain, the computational domain must be truncated which necessitates the imposition of numerical boundary condition at the artificial outflow boundary in order to close the system of equations. Unless a large computational domain is used for simulations so that detonation waves do not reach the artificial boundary before the final time is reached, waves reflected from the artificial boundary can be reflected back into the interior computational domain. The reflected waves will interact with the detonation waves and pollute the developing solution. Berenger, in [23], originally designed a Perfectly Matched Layer (PML) for Maxwell's equation to truncate an infinity or semi-infinity computational domain. It works as a relatively simple and efficient absorbing boundary condition for the wave problems. In the last few years, it was analyzed and developed in linearized and nonlinear Euler equations [24, 25, 26, 27] to absorb the outgoing waves and decrease the computational cost. In this study, we design an efficient and easily implemented $x$-direction PML for the unsteady reactive Euler equations to truncate the computational domain for two dimensional detonation waves. The additional cost of PML equation is negligible because the original Euler equation is only appended with an extra source term. The accuracy and efficiency of designed PML boundary condition are verified by comparing it with the characteristic, extrapolation, free stream boundary conditions and computing the pressure error against the reference solutions, which are obtained from a simulation with a large computational domain under the same grid resolution.

In this study of detonation wave simulations, we consider three classical examples listed in the following Table I, where $\gamma$ is the ratio of specific-heat, $E_{a}$ is the activation-energy parameter, $q_{0}$ is the heat-release parameter, $f$ is the overdrive factor.

We perturb the planar ZND wave by perturbing the vertical velocity $v$ in $y$ inside the half-reaction zone in the initial condition with a single-mode sinusoidal perturbation with small and large amplitude of perturbation. In the case $\left(H_{2}, E_{20}, f_{1.1}\right)$, solutions converge to steady state for high resolutions $\delta_{10}$ and $\delta_{20}$ (which denotes the resolution as 20 grid points per half-reaction zone), and 


\begin{tabular}{|c|c|c|c|c|c|}
\hline Cases studied & Abbreviation of cases & $\gamma$ & $q_{0}$ & $E_{a}$ & $f$ \\
\hline Small heat release/Low activation energy & $\left(H_{2}, E_{20}, f_{1.1}\right)$ & 1.2 & 2 & 20 & 1.1 \\
\hline Large heat release/Low activation energy & $\left(H_{50}, E_{10}, f_{1.2}\right)$ & 1.2 & 50 & 10 & 1.2 \\
\hline Large heat release/Large activation energy & $\left(H_{50}, E_{50}, f_{1.2}\right)$ & 1.2 & 50 & 50 & 1.2 \\
\hline
\end{tabular}

Table I: Cases of simulation and corresponding parameters.

the so-called regular rectangular modes are formed. In the more interesting case $\left(H_{50}, E_{10}, f_{1.2}\right)$, the corresponding one-dimensional case is an unstable case. However, it not only forms the rectangular mode in an earlier time but also generates the pockets of unburned gas in a later time. The structures of detonation waves becomes complicated at later time. In the most challenging case $\left(H_{50}, E_{50}, f_{1.2}\right)$, the one-dimensional flow with corresponding parameters is unstable case. The structure of detonation wave becomes very complicated when the transverse instabilities dominate the flow. The vorticity field structures are similar to display a strong two-dimensional incompressible flow turbulence [28].

Furthermore, we investigate the relation between the amplitude of perturbation wave and the behavior of detonation wave. Generally speaking, the larger amplitude releases larger amount of energy during the collision with shock front and that generates more small scale structures in the detonation waves. We also observe that the number of detonation cells in $y$-direction depends on the wave number of sinusoidal perturbation.

The paper is organized as follows. In Section 2, a brief introduction to WENO schemes for solving hyperbolic conservation laws is given. In Section 3, a brief introduction to two-dimensional detonation model with governing equations and its perturbed initial conditions is given. Different boundary conditions are briefly introduced and compared quantitatively and qualitatively in Section 3.2. Section 3.3 outlines the general framework of numerical schemes. Three numerical examples are simulated under different resolutions and various perturbation waves and their results are discussed in Section 4. Conclusion and remarks are given in Section 5.

\section{Weighted Essentially Non-Oscillation Schemes}

In this section we briefly describe the general framework of the $(2 r-1)$ order characteristics based weighted essentially non-oscillatory conservative finite difference scheme (WENO) for solving the system of hyperbolic conservation laws in the form

$$
\frac{\partial \mathbf{u}}{\partial t}+\nabla \cdot \mathbf{F}(\mathbf{u})=0
$$

Without loss of generalities, we will present the $(2 r-1)$ order conservative WENO finite difference 
scheme in one dimension. Explicit formula for fifth $(r=3)$ order scheme is given. Extension to higher order $(r>3)$ WENO scheme is straightforward.

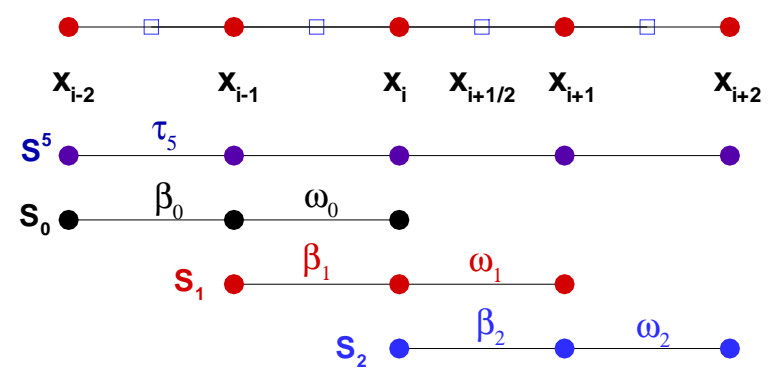

Figure 1: The computational uniform grid $x_{i}$ and the 5-points stencil $S^{5}$, composed of three 3-points substencils $S_{0}, S_{1}, S_{2}$, used for the fifth-order WENO reconstruction step.

Consider a uniform grid defined by the points $x_{i}=i \Delta x, i=0, \ldots, N$, which are called cell centers, with cell boundaries given by $x_{i+\frac{1}{2}}=x_{i}+\frac{\Delta x}{2}$, where $\Delta x$ is the uniform grid spacing (see Fig. 1). The semi-discretized form of (1) is transformed into the system of ordinary differential equations by method of line

$$
\frac{d u_{i}(t)}{d t}=-\left.\frac{\partial f}{\partial x}\right|_{x=x_{i}}, \quad i=0, \ldots, N,
$$

where $u_{i}(t)$ is a numerical approximation to the cell-averaged value $u\left(x_{i}, t\right)$.

To form the flux differences across the uniformly spaced cells, conservative finite-difference formulation for hyperbolic conservation laws requires high-order consistent numerical fluxes at the cell boundaries. We implicitly define the numerical flux function $h(x)$ as

$$
f(x)=\frac{1}{\Delta x} \int_{x-\frac{\Delta x}{2}}^{x+\frac{\Delta x}{2}} h(\xi) d \xi,
$$

such that the spatial derivative in (2) is exactly approximated by a conservative finite difference formula at the cell boundaries $x_{i \pm \frac{1}{2}}$,

$$
\frac{d u_{i}(t)}{d t}=\frac{1}{\Delta x}\left(h_{i+\frac{1}{2}}-h_{i-\frac{1}{2}}\right)
$$

where $h_{i \pm \frac{1}{2}}=h\left(x_{i \pm \frac{1}{2}}\right)$. High order polynomial interpolations to $h_{i \pm \frac{1}{2}}$ are computed using known cell-averaged values $f_{j}=f\left(x_{j}\right), j=i-r+1, \ldots, i, \ldots, i+r-1$.

The classical $(2 r-1)$ order WENO scheme uses $(2 r-1)$-points global stencil, which is subdivided into $r$ substencils $\left\{S_{0}, S_{1}, \ldots, S_{r-1}\right\}$ with each substencil containing $r$ grid points and a global 
stencils $S^{2 r-1}=\bigcup_{i=0}^{r-1} S_{i}$. For $r=3$, the 5 -points global stencil, hereafter named $S^{5}$, is subdivided into three 3 -points substencils $\left\{S_{0}, S_{1}, S_{2}\right\}$.

The $(2 r-1)$ degree polynomial approximation $\hat{f}_{i \pm \frac{1}{2}}=h_{i \pm \frac{1}{2}}+O\left(\Delta x^{2 r-1}\right)$ is built through the convex combination of the interpolated values $\hat{f}^{k}\left(x_{i \pm \frac{1}{2}}\right)$, in which $f^{k}(x)$ is the $r$ degree polynomial below, defined in each one of the substencils $S_{k}$ :

$$
\hat{f}_{i \pm \frac{1}{2}}=\sum_{k=0}^{r-1} \omega_{k} \hat{f}^{k}\left(x_{i \pm \frac{1}{2}}\right)
$$

where

$$
\hat{f}^{k}\left(x_{i+\frac{1}{2}}\right)=\hat{f}_{i+\frac{1}{2}}^{k}=\sum_{j=0}^{r-1} c_{k j} f_{i-k+j}, \quad i=0, \ldots, N .
$$

$\hat{f}_{i-\frac{1}{2}}^{k}$ can be found by symmetry. The $c_{k j}$ are Lagrangian interpolation coefficients (see [3]) and $\omega_{k}$ are normalized nonlinear weights (weights), which will be described below.

The regularity of the $(r-1)$ degree interpolation polynomial approximation $\hat{f}^{k}(x)$ at the substencil $S_{k}$ is measured by the lower order local smoothness indicators $\beta_{k}$, which are given by

$$
\beta_{k}=\sum_{l=1}^{r-1} \Delta x^{2 l-1} \int_{x_{i-\frac{1}{2}}}^{x_{i+\frac{1}{2}}}\left(\frac{d^{l}}{d x^{l}} \hat{f}^{k}(x)\right)^{2} d x .
$$

For $r=3$, the explicit expression of the $\beta_{k}$ in terms of the cell averaged values of $f(x), f_{i}$ are given by

$$
\begin{aligned}
& \beta_{0}=\frac{13}{12}\left(f_{i-2}-2 f_{i-1}+f_{i}\right)^{2}+\frac{1}{4}\left(f_{i-2}-4 f_{i-1}+3 f_{i}\right)^{2} \\
& \beta_{1}=\frac{13}{12}\left(f_{i-1}-2 f_{i}+f_{i+1}\right)^{2}+\frac{1}{4}\left(f_{i-1}-f_{i+1}\right)^{2} \\
& \beta_{2}=\frac{13}{12}\left(f_{i}-2 f_{i+1}+f_{i+2}\right)^{2}+\frac{1}{4}\left(3 f_{i}-4 f_{i+1}+f_{i+2}\right)^{2} .
\end{aligned}
$$

In the classical WENO scheme [1, 3] (WENO-JS), the normalized nonlinear weights $\omega_{k}$ and unnormalized nonlinear weights $\alpha_{k}$ in each substencil $S_{k}$, are defined as

$$
\omega_{k}=\frac{\alpha_{k}}{\sum_{l=0}^{r-1} \alpha_{l}}, \quad \alpha_{k}=\frac{d_{k}}{\left(\beta_{k}+\epsilon\right)^{p}}, \quad k=1, \ldots, r-1 .
$$

The parameter $\epsilon$ (typically $10^{-12}$ ) is used to avoid the division by zero in the denominator and power parameter $p=2$ is chosen to increase the difference of scales of distinct weights at nonsmooth parts of the solution. The coefficients $\left\{d_{0}, d_{1}, \ldots, d_{r-1}\right\}$ are called the ideal weights since 
they generate the $(2 r-1)$ order central upwind scheme using the $(2 r-1)$-points stencil when the solution is smooth. For $r=3$, the ideal weights are $\left\{d_{0}=\frac{3}{10}, d_{1}=\frac{3}{5}, d_{2}=\frac{1}{10}\right\}$.

The novel idea of the WENO-Z scheme (WENO-Z) $[5,29]$ is the modification of the $\beta_{k}$ with higher order information obtained from a global optimal order smoothness indicator, which is denoted here by $\tau_{2 r-1}$. This new global optimal order smoothness indicator $\tau_{2 r-1}$ is built using cell-averaged values in the whole $S^{2 r-1}$ stencil in the form of a linear combination of $\beta_{k}$, that is,

$$
\tau_{2 r-1}=\sum_{k=0}^{r-1} c_{k} \beta_{k},
$$

where $c_{k}$ are given constants (see [5, 29]).

The general definitions of the normalized and un-normalized nonlinear weights $\omega_{k}^{Z}$ and $\alpha_{k}^{Z}$, respectively, are

$$
\omega_{k}^{Z}=\frac{\alpha_{k}^{Z}}{\sum_{l=0}^{r-1} \alpha_{l}^{Z}}, \quad \alpha_{k}^{Z}=\frac{d_{k}}{\beta_{k}^{Z}}=d_{k}\left(1+\left(\frac{\tau_{2 r-1}}{\beta_{k}+\epsilon}\right)^{p}\right), \quad k=0, \ldots, r-1,
$$

where $p \geq 1$ is the power parameter, used to enhance the relative ratio between the smoothness indicators. For $r=3$, the global high order smoothness indicator $\tau_{5}$ is

$$
\tau_{5}=\left|\beta_{0}-\beta_{2}\right| .
$$

The numerical results in [5] confirmed the improved performance of the modified smoothness indicators over the WENO-JS scheme in computing a higher resolution solution when solving hyperbolic PDEs with discontinuous solutions.

\section{Governing Equations}

Two-dimensional unsteady reactive Euler equations for a perfect ideal gas coupled with irreversible chemical reaction in a conservation form is given by

$$
\frac{\partial \mathbf{Q}}{\partial t}+\nabla \cdot \overrightarrow{\mathbf{F}}=\mathbf{S}
$$

where $\overrightarrow{\mathbf{F}}=(\mathbf{F}, \mathbf{G})$ is the flux vector and

$$
\mathbf{Q}=\left[\begin{array}{c}
\rho \\
\rho u \\
\rho v \\
E \\
\rho f_{1}
\end{array}\right], \mathbf{F}=\left[\begin{array}{c}
\rho u \\
\rho u^{2}+P \\
\rho u v \\
(E+P) u \\
\rho f_{1} u
\end{array}\right], \mathbf{G}=\left[\begin{array}{c}
\rho v \\
\rho u v \\
\rho v^{2}+P \\
(E+P) v \\
\rho f_{1} v
\end{array}\right], \mathbf{S}=\left[\begin{array}{c}
0 \\
0 \\
0 \\
0 \\
\omega\left(T, f_{1}\right)
\end{array}\right] .
$$


where $\rho$ is density or mass density, $P$ is pressure, $u$ and $v$ are the $x$ - and $y$-component of velocities respectively. $f_{1}$ is the reactant mass fraction satisfying $0 \leq f_{1} \leq 1$, where $f_{1}=0$ describes the completely burned state and $f_{1}=1$ describes the unburned state.

The total specific energy $E$ is given by,

$$
E=\frac{P}{\gamma-1}+\frac{1}{2} \rho\left(u^{2}+v^{2}\right)+\rho f_{1} q_{0},
$$

and the source term $\omega\left(T, f_{1}\right)$ due to the chemical reaction is

$$
\omega\left(T, f_{1}\right)=-K \rho f_{1} e^{-E_{a} / T},
$$

where $\gamma$ is the ratio of specific-heat (assumed to be a constant here), $q_{0}$ is the heat-release parameter, $E_{a}$ is the activation-energy parameter, and $K$ is a pre-exponential factor that sets the spatial and temporal scales. The temperature is defined by

$$
T=P / \rho R,
$$

where $R$ is the specific gas constant (with a suitable normalization, $R=1$ in this study).

\subsection{Initial Conditions}

In the case of one-dimensional detonation wave the C-J velocity $D_{C J}$ and detonation velocity $D$ can be determined respectively with the given parameters $\gamma, q_{0}$, and the overdrive factor $f$. The flow variables $\rho, u$ and $P$ can be computed by specifying a given mass fraction $f_{1}$ profile. We refer to $[15,19]$ for details.

In this work we specify an exponential function as an initial profile of the mass fraction $f_{1}$. The corresponding one-dimensional typical ZND spatial profiles of different variables are shown in Fig. 2 which will be used as the setup of initial conditions of the detonation wave simulations.

In the case of two-dimensional problem, the initial condition in the $y$-component of velocity is given by a transversely sinusoidal perturbed planar ZND wave, i.e.,

$$
v(x, y, t=0)=\left\{\begin{array}{ll}
0 & x<x_{d}-W_{w} \\
A \sin \left(\frac{2 y \pi k}{L}\right) & x_{d}-W_{w} \leq x \leq x_{d} \\
0 & x>x_{d}
\end{array},\right.
$$

where $A, k$ and $L$ are the amplitude, wave number and wavelength of the sinusoidal perturbation of the detonation wave front and $W_{w}$ is the width of perturbation zone. $x_{d}$ is the initial location of the detonation wave front in the $x$-direction (the detonation waves travel from right to left with the frame of reference set on the detonation front). Since the problem is periodical in $y$-direction, $L$ is equal to the height of the domain in $y$-direction. In this study, $W_{w}=1$ and $L=10$ are used. 

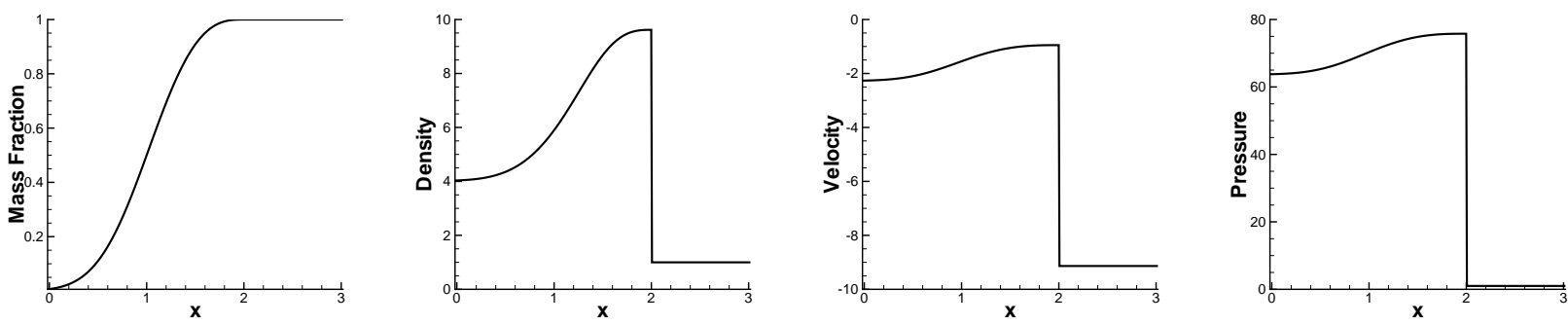

Figure 2: Initial profiles of mass fraction $f_{1}$, density $\rho$, velocity $u$ and pressure $P$ for an onedimensional ZND detonation wave.

\subsection{Boundary Conditions}

In this study, the problem is assumed to be periodical in the $y$-direction. Hence, periodical boundary condition is imposed in the $y$-direction. Since the frame of reference is set on the detonation front located inside the computational domain, the right boundary condition is set to be the initial free stream inflow condition.

The left boundary condition for the boundary/ghost cells, however, requires a more elaborate discussion as the detonation waves will travel to the left side of the domain if the nonlinear system of hyperbolic PDEs is integrated long enough in time. Depending on the length of the computational domain after truncating the physical domain to a finite size and the numerical boundary conditions imposed there, undesirable waves might reflect back into the computational domain and pollute the solution if the reflected wave is sufficiently strong. It should be noted that the left boundary is not a real physical boundary, like a solid wall, but an artificial one, created by the truncation of the physical domain into a computational domain. Theoretically, the grid points for the boundary/ghost points should satisfy the PDEs exactly. However, in order to close the discrete system, a numerical boundary condition must be imposed on the boundary/ghost points.

In this work, we will revisit three kinds of numerical boundary conditions, which have often been used in the published literature, and demonstrate their effectiveness in handling a truncated physical domain in the numerical simulation of detonation waves. An alternative approach based on the Perfectly Match Layer (PML) will then be proposed, described and compared with the other three approaches. It should be noted that, in this study, the problem of imposing left boundary condition for ghost cells is essentially one-dimensional.

The first approach for imposing numerical boundary condition is to assume the computational domain is sufficiently large such that no waves can reach the left truncated boundary at a given final time. In this case, the initial free stream condition can be naturally imposed. It guarantees the computed solution is free of pollution of reflected wave from the left computational domain boundary. Its solution can be used as reference solution when comparing different approaches in 
imposing left numerical boundary condition. The major downside of this approach is the cost of computing if the solution time is very large. Large number of grid points will be needed to maintain a specific grid resolution.

The second approach is to extrapolate the values of the ghost cells from the solution in the interior of the computational domain. This approach is easy and used often in the literature [30]. However, this approach will result in a well-posed problem only for supersonic flow and one can show that the flow at the left computational boundary is subsonic in this study.

The third approach is to employ the characteristic boundary condition for the boundary/ghost points by taking into account the direction of the propagating characteristic wave at the boundary. The outgoing characteristic waves will be allowed to move out of the computational domain and the incoming characteristic waves from free stream flow will be allowed to move into the computational domain. As discussed in the later Section 3.3, the eigensystem of the Euler equation with species with the right eigenvectors $\mathbf{R}$, left eigenvectors $\mathbf{R}^{-1}$ and eigenvalues $\boldsymbol{\Lambda}$ are provided.

The procedure of characteristic boundary condition can be summarized as follow: (ignoring the dependency in $y$ and assuming the boundary/ghost points are $x_{-r}, \ldots, x_{-1}$ for some $r>0$.)

1. Given the conservative variables $\mathbf{Q}$, the linearized eigensystem of the PDEs $\left(\mathbf{R}, \mathbf{R}^{-1}, \boldsymbol{\Lambda}\right)$ are computed at $x_{0}$, the first interior grid point.

2. The characteristic variable $\mathbf{W}$ is formed by projecting $\mathbf{Q}$ from the physical space onto the characteristic fields via the left eigenvectors as $\mathbf{W}=\mathbf{R}^{-1} \mathbf{Q}$ at all the grid points needed in the next step.

3. To impose the characteristic boundary condition, the characteristic variable $\mathbf{W}$ is modified according to the sign of the corresponding eigenvalues, that is, with $\overline{\mathbf{W}}\left(x_{0}\right)=\mathbf{W}\left(x_{0}\right)$ and $\overline{\mathbf{W}}\left(x_{1}\right)=\mathbf{W}\left(x_{1}\right)$,

$$
\bar{W}_{k}\left(x_{i-1}\right)=\left\{\begin{array}{ll}
2 \bar{W}_{k}\left(x_{i}\right)-\bar{W}_{k}\left(x_{i+1}\right) & \lambda_{k}\left(x_{0}\right) \geq 0 \\
W_{k}(x=-\infty) & \lambda_{k}\left(x_{0}\right)<0
\end{array}, \quad i=0, \ldots,-r+1,\right.
$$

where $W_{k}, \bar{W}_{k}$ and $\lambda_{k}$ are the $k_{-}$th component of the $\mathbf{W}$, updated characteristic variable $\overline{\mathbf{W}}$ and $\boldsymbol{\Lambda}$, respectively. Here we show the first order extrapolation of outgoing characteristic waves in a recursive manner.

4. An updated conservative variable $\mathbf{Q}$ can then be recovered by projecting $\overline{\mathbf{W}}$ from the characteristic fields back onto the physical space via the right eigenvectors as $\mathbf{Q}=\mathbf{R} \overline{\mathbf{W}}$.

The fourth approach is inspired from the idea of perfect match layer (PML) for unsteady Euler equations [27]. In PML, an extended domain (layer) is created to allow the transmission and damping of waves into the layer in order to minimize the reflection of the waves back into the 
computational domain. In essence, the conservative variables $\mathbf{Q}$ in the PML layer are separated into a time-independent steady part $\overline{\mathbf{Q}}_{p}$ and perturbed part $\mathbf{Q}^{\prime}$ as

$$
\mathbf{Q}=\overline{\mathbf{Q}}_{p}+\mathbf{Q}^{\prime}
$$

$\overline{\mathbf{Q}}_{p}$ is called a time-independent pseudo mean-flow in [27, 31] and satisfies the steady state Euler equation, that is,

$$
\frac{\partial \overline{\mathbf{Q}}_{p}}{\partial t}=\nabla \cdot \overrightarrow{\mathbf{F}}\left(\overline{\mathbf{Q}}_{p}\right)=0,
$$

where $\overrightarrow{\mathbf{F}}=(\mathbf{F}, \mathbf{G})$ is the flux vector in two dimensions.

Using (22), one can obtain the equation for $\mathbf{Q}^{\prime}$, that is,

$$
\frac{\partial \mathbf{Q}^{\prime}}{\partial t}+\nabla \cdot \delta \overrightarrow{\mathbf{F}}=0
$$

where $\delta \overrightarrow{\mathbf{F}}=\overrightarrow{\mathbf{F}}(\mathbf{Q})-\overrightarrow{\mathbf{F}}\left(\overline{\mathbf{Q}}_{p}\right)$.

In the frequency domain, it becomes

$$
(-i \omega) \tilde{\mathbf{Q}}^{\prime}+\nabla \cdot \widetilde{\delta \overrightarrow{\mathbf{F}}}=0
$$

where the tilde denotes a Laplace transformed quantity.

For clarity, we will present the method for a two dimensional problem. We shall denote $\delta \mathbf{F}=$ $\mathbf{F}(\mathbf{Q})-\mathbf{F}\left(\overline{\mathbf{Q}}_{p}\right)$ and $\delta \mathbf{G}=\mathbf{G}(\mathbf{Q})-\mathbf{G}\left(\overline{\mathbf{Q}}_{p}\right)$.

In the PML layer, by employing the change of complex variables

$$
x \longrightarrow x+\frac{i}{\omega} \int_{x_{p}}^{x} \sigma_{x}(\xi) d \xi, \quad y \longrightarrow y+\frac{i}{\omega} \int_{y_{p}}^{y} \sigma_{y}(\xi) d \xi,
$$

where $x_{p}\left(y_{p}\right)$ and $\sigma_{x}\left(\sigma_{y}\right)$ are the starting locations and smooth damping functions with $\sigma_{x}\left(x_{p}\right)=0$ $\left(\sigma_{y}\left(y_{p}\right)=0\right)$ of the PML layer in the $x(y)$-direction, respectively, one has

$$
(-i \omega) \tilde{\mathbf{Q}}^{\prime}+\frac{1}{1+i \frac{\sigma_{x}}{\omega}} \frac{\widetilde{\partial \delta \mathbf{F}}}{\partial x}+\frac{1}{1+i \frac{\sigma_{y}}{\omega}} \frac{\widetilde{\partial \delta \mathbf{G}}}{\partial y}=0 .
$$

By splitting $\tilde{\mathbf{Q}}^{\prime}$ into $\tilde{\mathbf{Q}}_{1}^{\prime}$ and $\tilde{\mathbf{Q}}_{2}^{\prime}$ which are the flow variables in $x$ - and $y$-directions respectively, (26) can be written as

$$
\begin{aligned}
& (-i \omega) \tilde{\mathbf{Q}}_{1}^{\prime}+\frac{1}{1+i \frac{\sigma_{x}}{\omega}} \frac{\widetilde{\partial \delta \mathbf{F}}}{\partial x}=0 \\
& (-i \omega) \tilde{\mathbf{Q}}_{2}^{\prime}+\frac{1}{1+i \frac{\sigma_{y}}{\omega}} \frac{\widetilde{\partial \delta \mathbf{G}}}{\partial y}=0
\end{aligned}
$$


where $\tilde{\mathbf{Q}}^{\prime}=\tilde{\mathbf{Q}}_{1}^{\prime}+\tilde{\mathbf{Q}}_{2}^{\prime}$.

By rearranging the equations above in the time domain, one has

$$
\begin{aligned}
& \frac{\partial \mathbf{Q}_{1}^{\prime}}{\partial t}+\sigma_{x} \mathbf{Q}_{1}^{\prime}+\frac{\partial \delta \mathbf{F}}{\partial x}=0 \\
& \frac{\partial \mathbf{Q}_{2}^{\prime}}{\partial t}+\sigma_{y} \mathbf{Q}_{2}^{\prime}+\frac{\partial \delta \mathbf{G}}{\partial y}=0 .
\end{aligned}
$$

The two equations above are for absorbing $\mathbf{Q}^{\prime}$ in the PML layer. By adding them together, an equation for $\mathbf{Q}^{\prime}$ can be formed, namely,

$$
\frac{\partial \mathbf{Q}^{\prime}}{\partial t}+\sigma_{x}\left(\mathbf{Q}^{\prime}-\mathbf{Q}_{2}^{\prime}\right)+\frac{\partial \delta \mathbf{F}}{\partial x}+\sigma_{y}\left(\mathbf{Q}^{\prime}-\mathbf{Q}_{1}^{\prime}\right)+\frac{\partial \delta \mathbf{G}}{\partial y}=0
$$

Since $\overline{\mathbf{Q}}_{p}$ satisfies the steady Euler equation (22), the PML equation for $\mathbf{Q}$ can be written as

$$
\begin{aligned}
\frac{\partial \mathbf{Q}}{\partial t}+\nabla \cdot \overrightarrow{\mathbf{F}}+\sigma_{x}\left(\mathbf{Q}-\overline{\mathbf{Q}}_{p}-\mathbf{Q}_{2}^{\prime}\right)+\sigma_{y}\left(\mathbf{Q}-\overline{\mathbf{Q}}_{p}-\mathbf{Q}_{1}^{\prime}\right) & =0, \\
\frac{\partial \mathbf{Q}_{2}^{\prime}}{\partial t}+\sigma_{y} \mathbf{Q}_{2}^{\prime}+\frac{\partial \delta \mathbf{G}}{\partial y} & =0 .
\end{aligned}
$$

In this work, PML is not needed in the periodical $y$-direction. Therefore, one can neglect (33) and assume $\sigma_{y}=0$ and $\mathbf{Q}_{2}^{\prime}=0$ in the implementation of the above PML in our study. We also assume $\overline{\mathbf{Q}}_{p}=\mathbf{Q}_{0}$ which is the initial condition for steady ZND solution inside the PML layer. We refer to $[27]$ for more details.

To illustrate the efficiency and accuracy of $(32)$, we consider the case $\left(H_{2}, E_{20}, f_{1.1}\right)$ with following parameters (see Section 4.1 for details)

$$
q_{0}=2, \quad E_{a}=20, \quad f=1.1,
$$

as proposed in $[16,32]$ as a classical example of two-dimensional detonation wave. The computational domain is $(x, y)=[0,230] \times[-5,5]$ and the solution is defined as reference solution since no flow structure has reached the left end of the boundary at $x=0$ at the final time. With the implementation of PML boundary condition, the computational domain is set to be $(x, y)=[100,230] \times[-5,5]$, and PML layer is $\left(x_{a}, x_{b}\right)=[100,106]$ and detonation front is initially located at $x_{d}=225$. The PML layer damping function $\sigma_{x}$ is defined as

$$
\sigma_{x}=\left\{\begin{array}{ll}
\alpha\left|\frac{x-x_{b}}{x_{a}-x_{b}}\right|^{\beta} & x_{a} \leq x<x_{b} \\
0 & \text { else }
\end{array},\right.
$$

where $\alpha=60$ and $\beta=4$.

The comparison of the pressure between the four kinds of boundary condition and the reference solution is shown in Fig. 3. Reflection of waves from the left boundary are observed in every kinds of 
boundary condition discussed above. The PML boundary condition has the minimal pressure wave reflection from the left computational boundary with maximum absolute error less than $2 \times 10^{-5}$ while the error of other three kinds of boundary condition can be up to $2 \times 10^{-2}$. Hence, we will employ the PML boundary condition in this study of detonation waves.

\section{Characteristics}

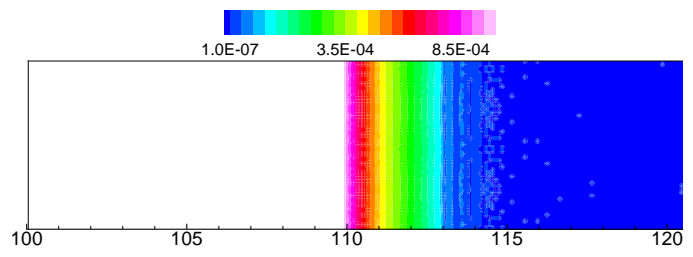

Free stream

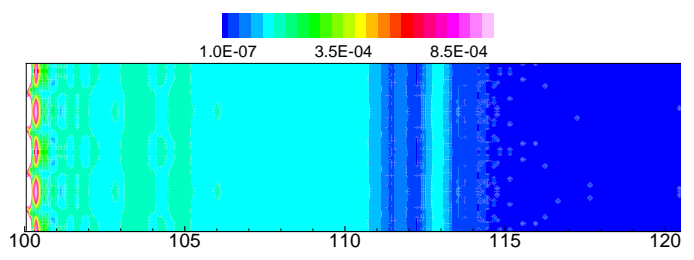

\section{Extrapolation}

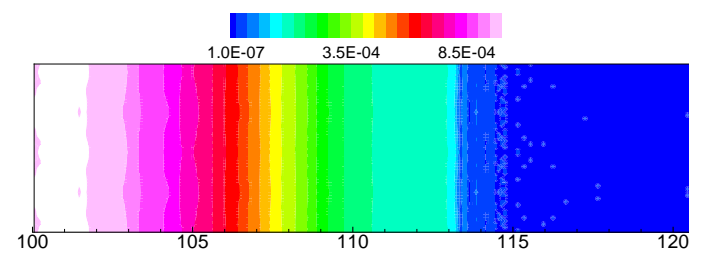

PML

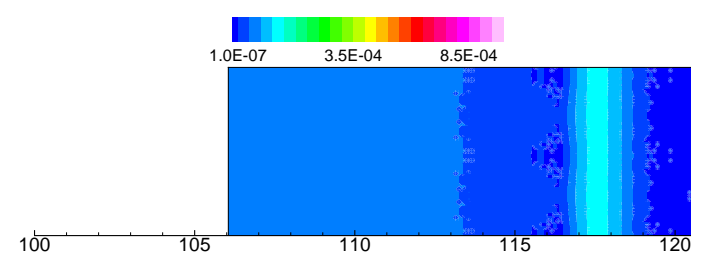

Figure 3: $\left(H_{2}, E_{20}, f_{1.1}\right)$ : Absolute error in pressure of the characteristic, zero-order extrapolation, free stream and PML boundary conditions when compared with the reference solution computed from a large computational domain.

\subsection{Numerical Methods}

We approximate the inviscid flux, via the well-known fifth order characteristic-based weighted essentially non-oscillatory conservative finite difference scheme (WENO). Following $[1,3,5]$, the hyperbolicity of the Euler equations admits a complete set of right and left eigenvectors for the Jacobian of the system.

By defining the two dimensional unit outward normal vector $\mathbf{n}=\left(n_{x}, n_{y}\right)=(\cos \theta, \sin \theta)$, where $\theta=\tan ^{-1}\left(n_{y} / n_{x}\right)$, and the rotation matrix

$$
\mathbf{T}=\left[\begin{array}{ccccc}
1 & 0 & 0 & 0 & 0 \\
0 & n_{x} & n_{y} & 0 & 0 \\
0 & -n_{y} & n_{x} & 0 & 0 \\
0 & 0 & 0 & 1 & 0 \\
0 & 0 & 0 & 0 & 1
\end{array}\right], \quad \mathbf{T}^{-1}=\left[\begin{array}{ccccc}
1 & 0 & 0 & 0 & 0 \\
0 & n_{x} & -n_{y} & 0 & 0 \\
0 & n_{y} & n_{x} & 0 & 0 \\
0 & 0 & 0 & 1 & 0 \\
0 & 0 & 0 & 0 & 1
\end{array}\right]
$$


the Euler system has Jacobian in general form as

$$
\mathbf{A}=\left(\mathbf{F}_{J}, \mathbf{G}_{J}\right) \cdot \mathbf{n},
$$

where $\mathbf{F}_{J}$ and $\mathbf{G}_{J}$ are Jacobian matrices of fluxes of $\mathbf{F}$ and $\mathbf{G}$, respectively. One can then find the right eigenvectors $\mathbf{R}(\mathbf{Q})$, left eigenvectors $\mathbf{R}^{-1}(\mathbf{Q})$ and eigenvalues $\boldsymbol{\Lambda}(\mathbf{Q})$ of $\mathbf{F}_{J}$. In the Cartesian frame, the eigensystem is a function of $\mathbf{Q}$.

By the rotational invariance property of Euler equations [33], the eigensystem of equation (16) can be represented generally as

$$
\mathbf{A}=\hat{\mathbf{R}} \hat{\mathbf{\Lambda}} \hat{\mathbf{R}}^{-1},
$$

where $\hat{\mathbf{Q}}=\mathbf{T} \mathbf{Q}, \hat{\mathbf{R}}=\mathbf{T}^{-1} \mathbf{R}(\hat{\mathbf{Q}}), \hat{\mathbf{R}}^{-1}=\mathbf{R}^{-1}(\hat{\mathbf{Q}}) \mathbf{T}, \hat{\mathbf{\Lambda}}=\mathbf{\Lambda}(\hat{\mathbf{Q}})$. In a rotational frame, the eigensystem is a function of $\hat{\mathbf{Q}}$.

Hence, the Jacobian matrix of system (16) becomes,

$$
\mathbf{A}=\left(\begin{array}{rrr}
0 & n_{x} \\
\frac{1}{2}(\gamma-3) u^{2} n_{x}-u v n_{y} & (3-\gamma) u n_{x}+v n_{y} \\
\frac{1}{2}(\gamma-3) v^{2} n_{y}-u v n_{x} & (1-\gamma) u n_{y}+v n_{x} \\
\left((\gamma-1) \mathbf{u}^{2}-h\right) \mathbf{u} \cdot \mathbf{n} & \left.\left(h-u^{2}(\gamma-1)\right) n_{x}+(1-\gamma u v) n_{y}\right) \\
-f_{1} \mathbf{u} \cdot \mathbf{n} & f_{1} n_{x}
\end{array}\right.
$$

The right and left eigenvector matrices of $\mathbf{A}$ are

$$
\hat{\mathbf{R}}=\left(\begin{array}{rrrrrr}
1 & 1 & 1 & 0 & 1 \\
u+c n_{x} & u-c n_{x} & (\mathbf{u} \cdot \mathbf{n}) n_{x} & -n_{y} & (\mathbf{u} \cdot \mathbf{n}) n_{x} \\
v+c n_{y} & v-c n_{y} & (\mathbf{u} \cdot \mathbf{n}) n_{y} & n_{x} & (\mathbf{u} \cdot \mathbf{n}) n_{y} \\
h+c \mathbf{u} \cdot \mathbf{n} & h-c \mathbf{u} \cdot \mathbf{n} & \frac{\zeta}{2} & \mathbf{u} \cdot \mathbf{k} & 0 \\
f_{1} & f_{1} & 0 & 0 & -\frac{\zeta}{2 q_{0}}
\end{array}\right)
$$




$$
\hat{\mathbf{R}}^{-1}=\tilde{c}\left(\begin{array}{rrr}
\frac{\mathbf{u}^{2}}{2}-\frac{c \mathbf{u} \cdot \mathbf{n}}{\gamma-1} & \frac{c}{\gamma-1} n_{x}-u & \frac{c}{\gamma-1} n_{y}-v \\
\frac{\mathbf{u}^{2}}{2}+\frac{c \mathbf{u} \cdot \mathbf{n}}{\gamma-1} & -\frac{c}{\gamma-1} n_{x}-u & -\frac{c}{\gamma-1} n_{y}-v \\
2\left(h-\mathbf{u}^{2}\right)-\frac{4 f_{1} q_{0}(\mathbf{u} \cdot \mathbf{n})^{2}}{\zeta} & 2 u+\frac{4 f_{1} q_{0} u}{\zeta} & 2 v+\frac{4 f_{1} q_{0} v}{\zeta} \\
-(\mathbf{u} \cdot \mathbf{k}) \mathbf{u}^{2} & 2 u(\mathbf{u} \cdot \mathbf{k})-\frac{n_{y}}{\tilde{c}} & 2 v(\mathbf{u} \cdot \mathbf{k})+\frac{n_{x}}{\tilde{c}} \\
\frac{2 f q_{0} \mathbf{u}^{2}}{\zeta} & -\frac{4 f q_{0} u}{\zeta} & -\frac{4 f q_{0} v}{\zeta}
\end{array}\right.
$$

$$
\left.\begin{array}{rr}
1 & -q_{0} \\
1 & -q_{0} \\
-2-\frac{4 f_{1} q_{0}}{\zeta} & \frac{4 q_{0}\left(h-(\mathbf{u} \cdot \mathbf{k})^{2}\right)}{\zeta} \\
-2 \mathbf{u} \cdot \mathbf{k} & 2 q_{0} \mathbf{u} \cdot \mathbf{k} \\
\frac{4 f_{1} q_{0}}{\zeta} & \frac{2 q_{0}\left(\mathbf{u}^{2}-2 h\right)}{\zeta}
\end{array}\right)
$$

and their corresponding eigenvalues are

$$
\lambda_{1}=\mathbf{u} \cdot \mathbf{n}+c, \lambda_{2}=\mathbf{u} \cdot \mathbf{n}-c, \lambda_{3}=\lambda_{4}=\lambda_{5}=\mathbf{u} \cdot \mathbf{n} .
$$

Here, $h=(E+P) / \rho$ and $c=\sqrt{\gamma P / \rho}$ are the specific enthalpy and sound speed, respectively. Also, we define $\tilde{c}=(\gamma-1) /\left(2 c^{2}\right), \mathbf{u} \cdot \mathbf{n}=u n_{x}+v n_{y}, \mathbf{u} \cdot \mathbf{k}=-u n_{y}+v n_{x}, \mathbf{u}^{2}=\mathbf{u} \cdot \mathbf{u}=(\mathbf{u} \cdot \mathbf{n})^{2}+(\mathbf{u} \cdot \mathbf{k})^{2}=$ $u^{2}+v^{2}, \zeta=(\mathbf{u} \cdot \mathbf{n})^{2}-(\mathbf{u} \cdot \mathbf{k})^{2}$.

The approximated eigenvalues and eigenvectors are obtained via the Roe linearized Riemann solver. The first order global Lax-Friedrichs flux is used as the low order building block for the high order reconstruction step of the WENO scheme. After projecting the positive and negative fluxes on the characteristic fields via the left eigenvectors, the high order WENO reconstruction step is applied to obtain the high order approximation at the cell boundaries using the surrounding cell-centered values, which are then projected back into the physical space via the right eigenvectors and added together to form a high order numerical flux at the cell-interfaces. The conservative difference of the reconstructed high order fluxes can then be computed for inviscid flux.

The resulting system of ordinary differentiation equations ODE (4) is advanced in time via the third order TVD Runge-Kutta scheme. The CFL condition is set to be CFL $=0.45$ in the numerical experiments performed in this study. We refer to $[3,5]$ for further details on the WENO algorithm for solving the hyperbolic conservation laws. 


\section{Numerical Experiments and Discussions}

In this section, the fifth order WENO-Z scheme is employed to simulate three classical twodimensional examples of detonation waves, all of which have been studied previously, albeit using different techniques.

\subsection{Small heat release and low activation energy}

In the case $\left(H_{2}, E_{20}, f_{1.1}\right)$, parameters are chosen in Table I whose corresponding stiffness coefficient and detonation front speed are $K=1134363.64$ and $D=2.04$ respectively. The one-dimensional flow with corresponding parameters is a linearly stable case because there is no linearly unstable longitudinal modes $[34,35]$. The parameters of perturbation wave are $(k=4, A=0.1)$. The computational domain is set to be $(x, y)=[100,230] \times[-5,5]$, and PML layer is $(x, y)=[100,106] \times$ $[-5,5]$ and detonation front is initially located at $x_{d}=225$.

Fig. 4 (a) and (b) display mesh refinement study of WENO-Z scheme, peak pressure and detonation front speed are measured in reaction zone at time $t=100$. The computed solution converges to steady state solution as grid resolution increases, and it agrees well with [36] and our previous work [19]. The temporal evolution of the peak pressure $P_{m}(t)$ in reaction zone under grid resolutions $\delta_{5}$, $\delta_{10}$ and $\delta_{20}$ are shown in Fig. 4 (c). In low grid resolution $\delta_{5}$, the scheme does not reach the steady state solution. As high grid resolutions $\delta_{10}$ and $\delta_{20}$, both results converge to steady state solution for time $t>30$.

(a) Peak pressure $P_{m}(t)$

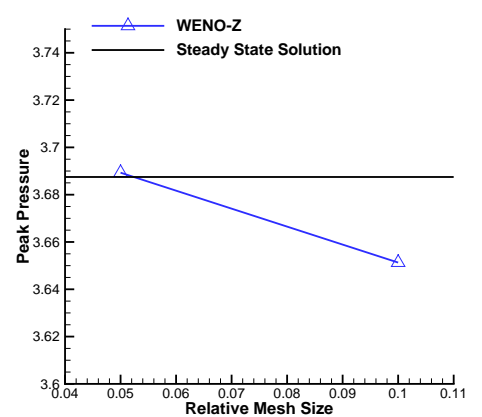

(b) CJ Speed $D_{C J}$

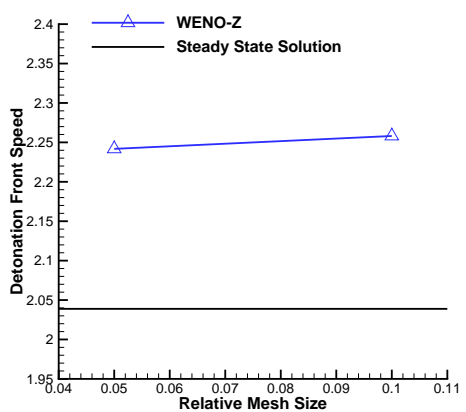

(c) Peak Pressure $P_{m}(t)$

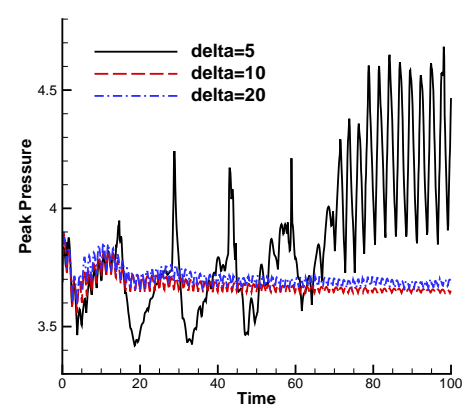

Figure 4: $\left(H_{2}, E_{20}, f_{1.1}\right)$ : (Color online) Mesh study of (a) peak pressure $P_{m}(t)$ and (b) detonation front speed $D_{C J}$ at time $t=100$. (c) $P_{m}(t)$ with grid resolutions $\delta_{5}, \delta_{10}$ and $\delta_{20}$.

Pressure and vorticity contour with grid resolutions $\delta_{10}$ and $\delta_{20}$ at time $t=30$ are given in the Fig. 5. The flow variables generated regular cellular structures which are due to the large amount 
of energy being released during the collisions between the perturbation waves and shock fronts. They are in very good agreement with the results presented in the literature [16, 32]. Furthermore, the results of high grid resolution simulations capture more small scale structures than the low grid resolution one in the Fig. 5.
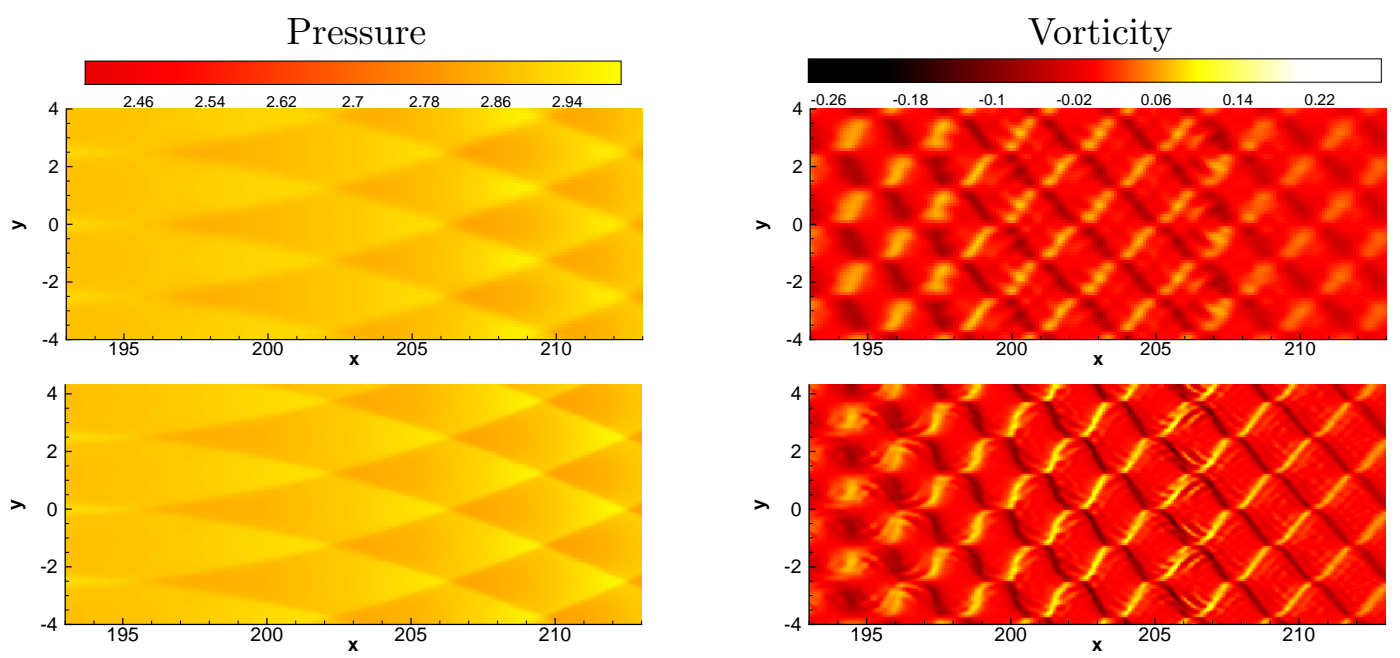

Figure 5: $\left(H_{2}, E_{20}, f_{1.1}\right)$ : (Color online) The profiles of pressure and vorticity at time $t=30$ with grid resolutions $\delta_{10}$ (first row) and $\delta_{20}$ (second row).

To explore the relation between amplitude of perturbation wave $A$ and structures of the detonation wave, we increase the amplitude from $A=0.1$ to $A=0.5$ and show the temporal evolution of the peak pressure $P_{m}(t)$ at time $t=100$ and pressure at time $t=10$ with grid resolution $\delta_{20}$. In the Fig. 6 (a), initially, large amplitude case generates high peak pressure in reaction zone as expected because more energy is being released when the shock fronts interacted with the stronger perturbation wave. Because of the stability in this case, perturbations decay with time that indicates the steady solution is hydrodynamically stable and probably unique [11]. In Fig. 6 (b), the cellular structures are similar for both cases, and results of large amplitude are better developed than those of small amplitude. In Fig. 6 (c) and (d), we show the pressure structure of detonation front for large amplitude $A=0.5$ and grid resolution $\delta_{20}$ before and after triple points collision. The pressure structure of detonation front agrees well with $[11,32]$.

\subsection{Large heat release and low activation energy}

The case $\left(H_{50}, E_{10}, f_{1.2}\right)$ has no prediction of regular cell structures from the theory of instability based on geometric acoustics [32] and the corresponding one-dimensional flow is unstable. The 
(a) $P_{m}(t)$

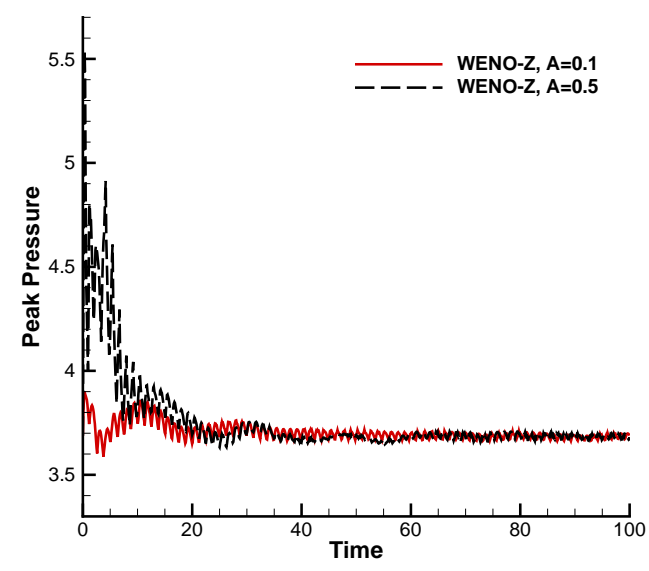

(c) Pressure before collision

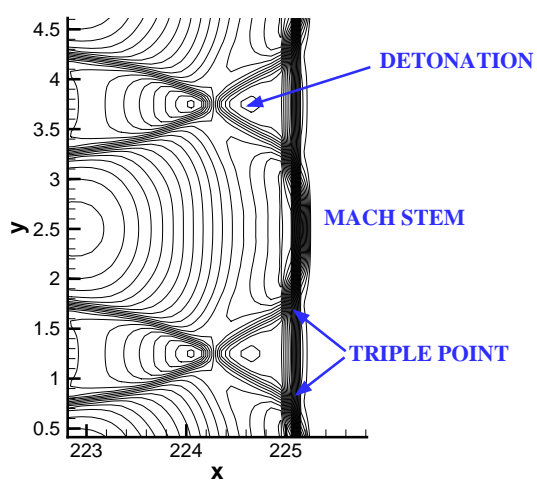

(b) Pressure
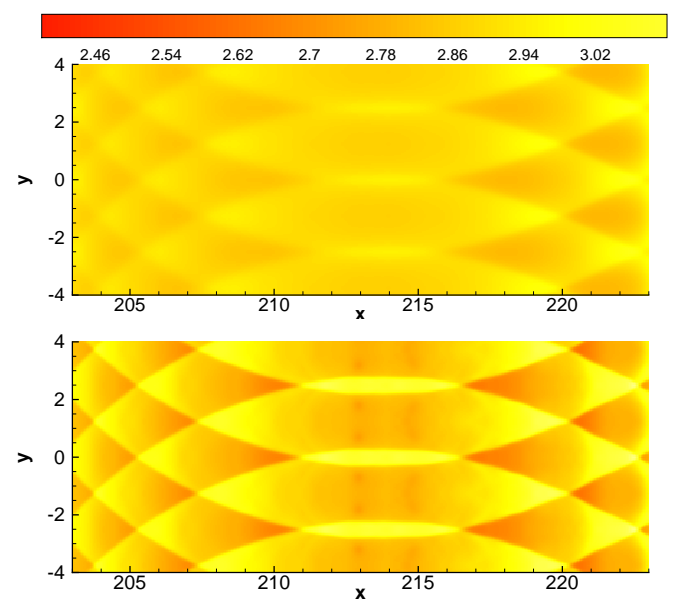

(d) Pressure after collision

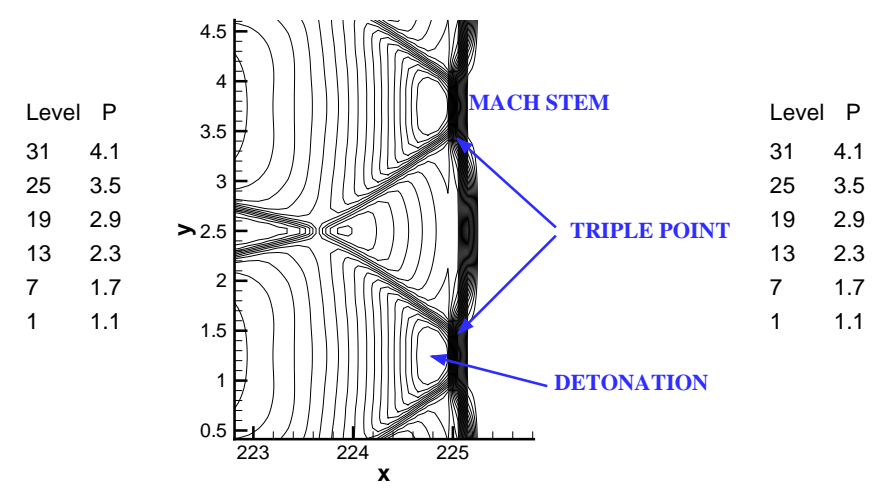

Figure 6: $\left(H_{2}, E_{20}, f_{1.1}\right)$ : (Color online) (a) The peak pressure $P_{m}(t)$ for perturbation amplitudes $A=0.1$ and $A=0.5$. (b) Pressure contour of $A=0.1$ (upper) and $A=0.5$ (lower). (c) Pressure contour before the triple points collision. (d) Pressure contour after the triple points collision. The grid resolution is $\delta_{20}$. 
stiffness coefficient and detonation front speed are $K=3.124$ and $D=2.04$ respectively. The computational domain is $(x, y)=[300,410] \times[-5,5]$ and PML layer is $(x, y)=[300,306] \times[-5,5]$. The ZND wave is initially located at $x_{d}=400$.

In Fig. 7, we show the comparative results of the temporal evolution of peak pressure $P_{m}(t)$ at time $t=50$ with different grid resolutions $\delta_{5}, \delta_{10}, \delta_{20}$ and various perturbation waves $(k=2, A=$ $0.5),(k=2, A=1.0),(k=3, A=0.5)$. The grid resolution study of $P_{m}(t)$ shows that WENO-Z scheme with lower grid resolution $\delta_{5}$ cannot capture the small scale structures and the natural phenomena of detonation wave. In contrast, higher peak pressures $P_{m}(t)$ with regular pattern are captured with high grid resolution $\delta_{10}, \delta_{20}$ at late time. The peak pressure also increases to a higher value when we perturbed the initial condition with larger amplitude $A=1.0$. Furthermore, we record the temporal evolution of the pressure $P$ and velocity $u$ at location $(x, y)=(355,0)$ which are shown in Fig. 8.
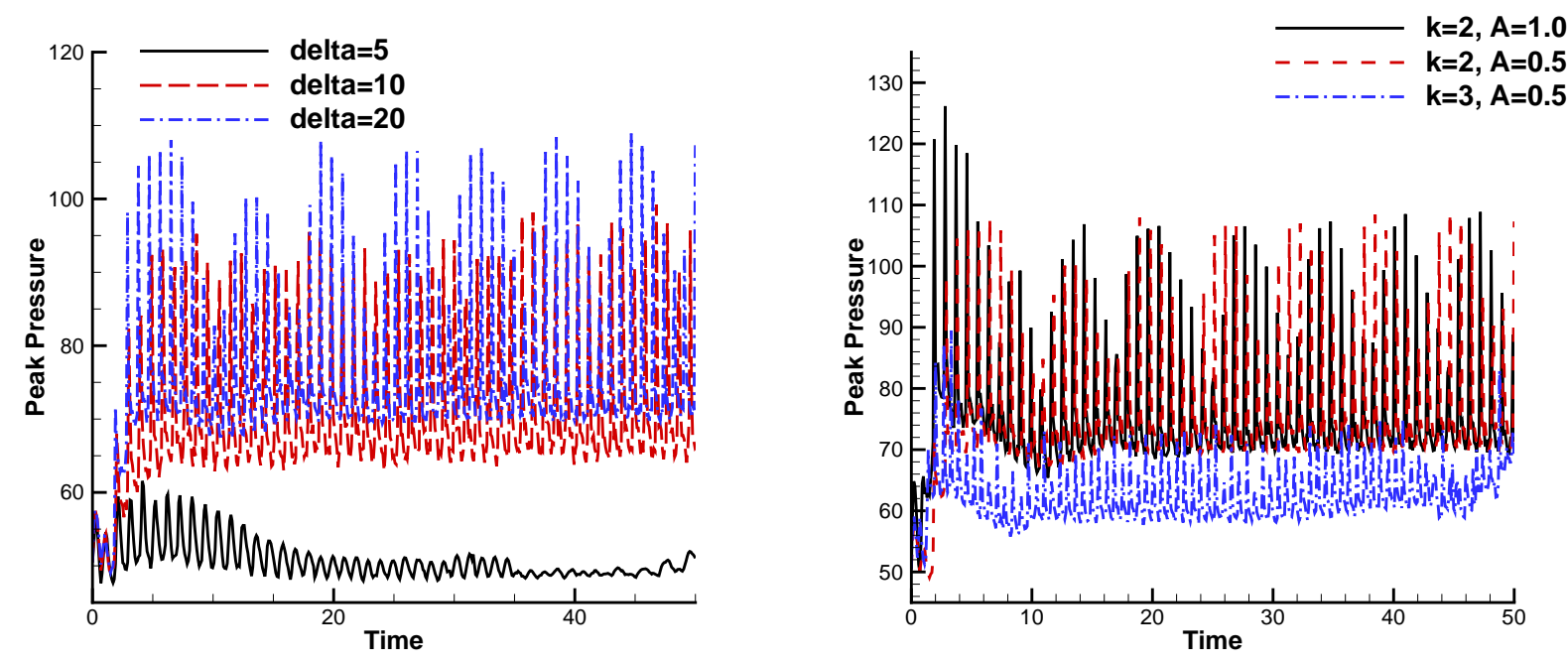

Figure 7: $\left(H_{50}, E_{10}, f_{1.2}\right)$ : (Color online) The temporal evolution of peak pressure $P_{m}(t)$ with (Left) $\delta_{5}, \delta_{10}, \delta_{20}$ under the perturbation wave parameters $(k=2, A=0.5)$ and (Right) three sets of perturbation wave parameters $(k, A)$. The grid resolution is $\delta_{20}$.

The contours of flow variables computed by WENO-Z scheme at time $t=10$ with grid resolution $\delta_{20}$ are shown in Fig. 9 whose structures agree well with those in the literature [16, 32]. We observe that two and three detonation cells in $y$-direction are formed in the case of $k=2$ and $k=3$ respectively. It agrees well with the conclusion that the final number of cells depends on the specific initial perturbation. We refer to [32] for details.

The detonation structures in the flow variables become more complicated than those generated in the previous case of small heat release and low activation energy, such as, the regular rectangle 

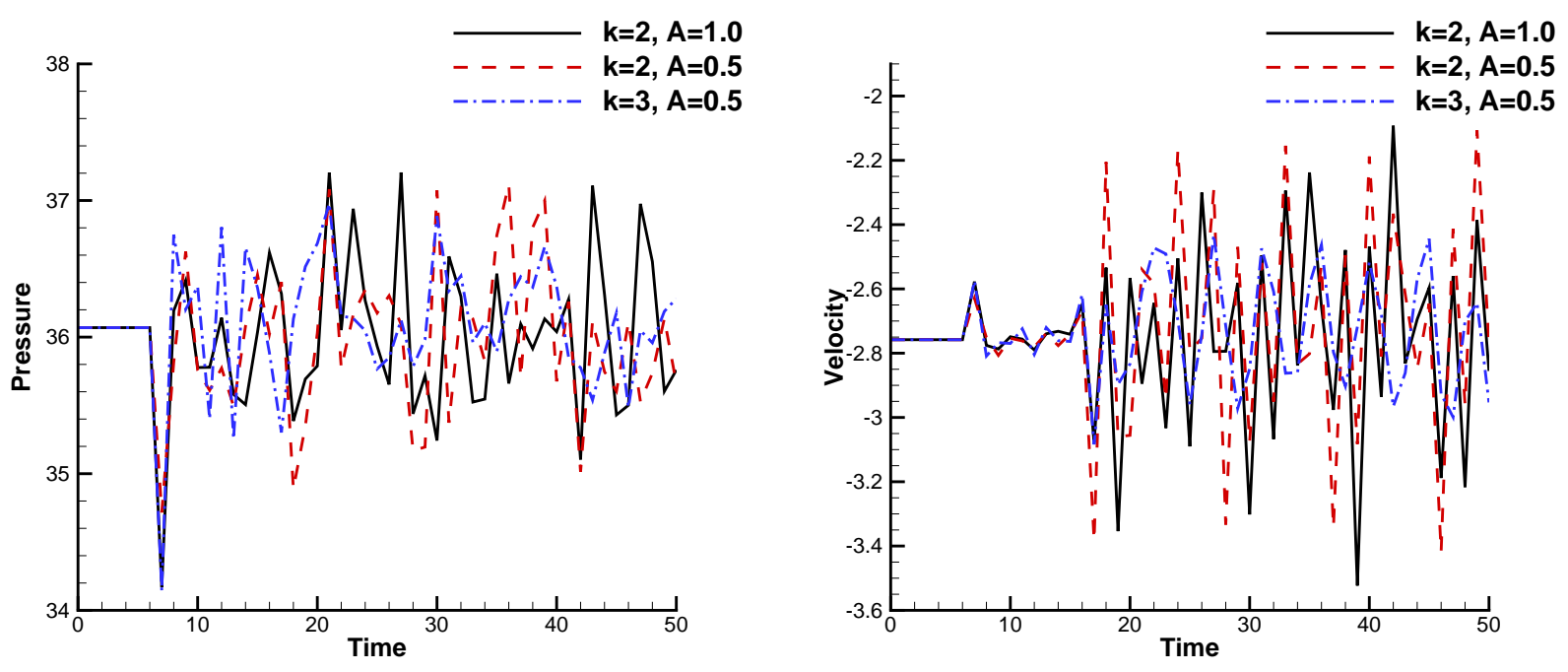

Figure 8: $\left(H_{50}, E_{10}, f_{1.2}\right)$ : (Color online) (Left) Pressure $P$ and (Right) Velocity $u$ with grid resolution $\delta_{20}$ at locations $(x, y)=(355,0)$.

structure in the pressure disappear. Furthermore, the vortex sheet are well developed into regular pairs from two colliding triple points when they detached from front. The mushroom shaped vortex rollup structures are formed in vorticity, temperature and mass fraction. The structures in the results of larger amplitude $A=1.0$ are more complicated than those of smaller amplitude $A=0.5$, such as, the vortex rolls up earlier with larger amplitude $A=1.0$ than the smaller amplitude $A=0.5(k=2)$. We also observe that, the vortex starts rolling up further upstream at location $x=375$ with $k=2$ but not with $k=3(A=0.5)$.

\subsection{Large heat release and large activation energy}

In this section we present numerical results of a challenging case $\left(H_{50}, E_{50}, f_{1.2}\right)$, which is the unstable ZND wave at arbitrarily short wavelengths [16, 32]. The corresponding stiffness coefficient and detonation front speed of this system is $K=871.42$ and $D=7.4594$ respectively. The computational domain is $(x, y)=[300,410] \times[-5,5]$ and PML layer is $(x, y)=[300,306] \times[-5,5]$. The ZND wave is initially located at $x_{d}=400$.

The comparative profiles of pressure, temperature, vorticity and mass fraction between two perturbation cases $(k=2, A=0.1)$ and $(k=2, A=0.2)$ computed by WENO-Z scheme with grid resolution $\delta_{20}$ at times $t=10,15,20$ are present in the Fig. 10 and Fig. 11. The result agrees well with those in the literature $[16,32]$. The structures of detonation wave becomes increasingly 

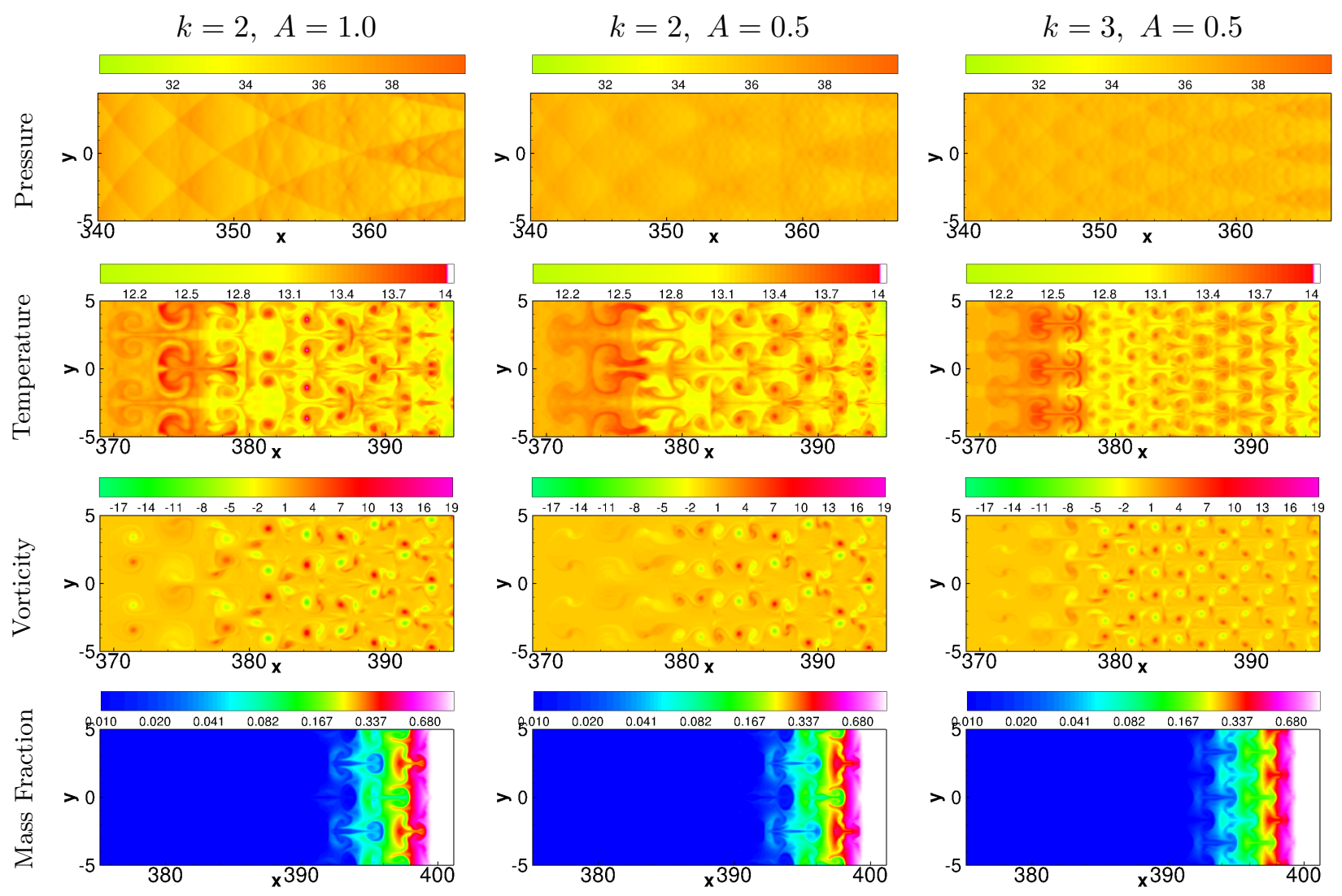

Figure 9: $\left(H_{50}, E_{10}, f_{1.2}\right)$ : (Color online) Contours of the pressure, temperature, vorticity and mass fraction with grid resolution $\delta_{20}$ under the perturbation waves with parameters $(k=2, A=$ $1.0),(k=2, A=0.5)$ and $(k=3, A=0.5)$. 
chaotic with time increases. At the early time $t=10$, two regular detonation cellular structures are formed and the vortices are well developed and symmetrically distributed in the wake of the front. The detonation wave structure starts becoming irregular at time $t=15$ due to the material burns fast resulting in high over-pressures. Meanwhile, strong pockets of unburned gas are constantly generated and subsequently burnt. The detonation wave structure becomes chaotic at time $t=20$ when the transverse instabilities grow strong and dominate the flow. Furthermore, in both cases, the vorticity generates similar two-dimensional incompressible turbulence at $t=20$ as reported in [28]. In the case of large amplitude $(k=2, A=0.2)$, more small scale structures in the detonations wave are generated due to larger amount of energy being released during the collision with shock front more than those in the case of small amplitude $(k=2, A=0.1)$.
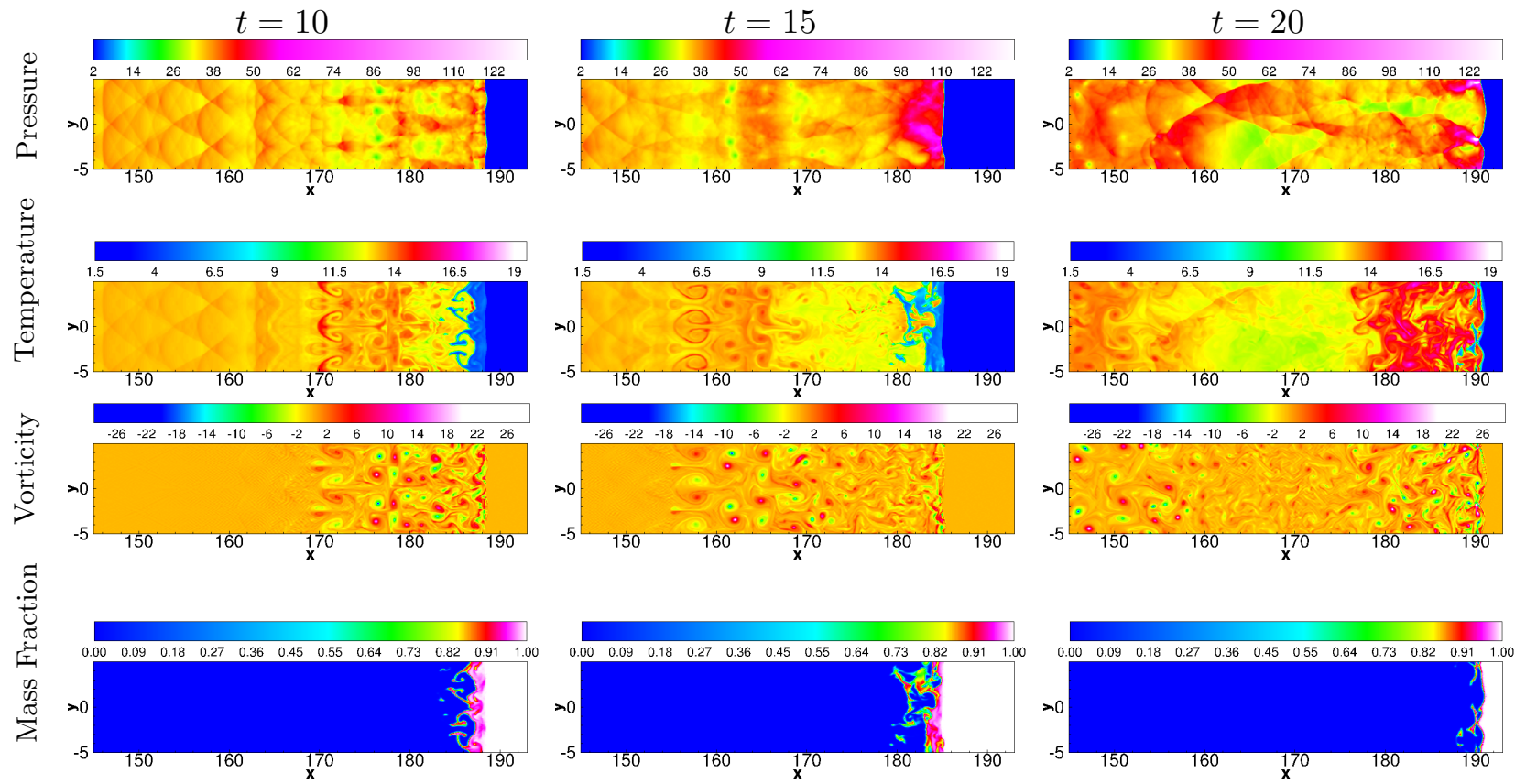

Figure 10: $\left(H_{50}, E_{50}, f_{1.2}\right):$ (Color online) Contours of the pressure, temperature, vorticity and mass fraction at times $t=10,15,20$ with grid resolution $\delta_{20}$. The parameters used for the perturbation waves are $(k=2, A=0.1)$.

We choose two symmetric locations, namely $(185,2.5)$ and $(185,-2.5)$, to record the evolution of pressure $P$ and velocity $u$ in the perturbation cases $(k=2, A=0.1)$ and $(k=2, A=0.2)$ as shown in Fig. 12. At the early time, their evolution are identical, the symmetry property is gradually lost due to the irregularity of detonation as time increases. And larger amplitude $(k=2, A=0.2)$ case loses the flow symmetry earlier than smaller amplitude $(k=2, A=0.1)$ case, which conforms to the theory that strong turbulence flow contributes the irregularity of cellar pattern early. 

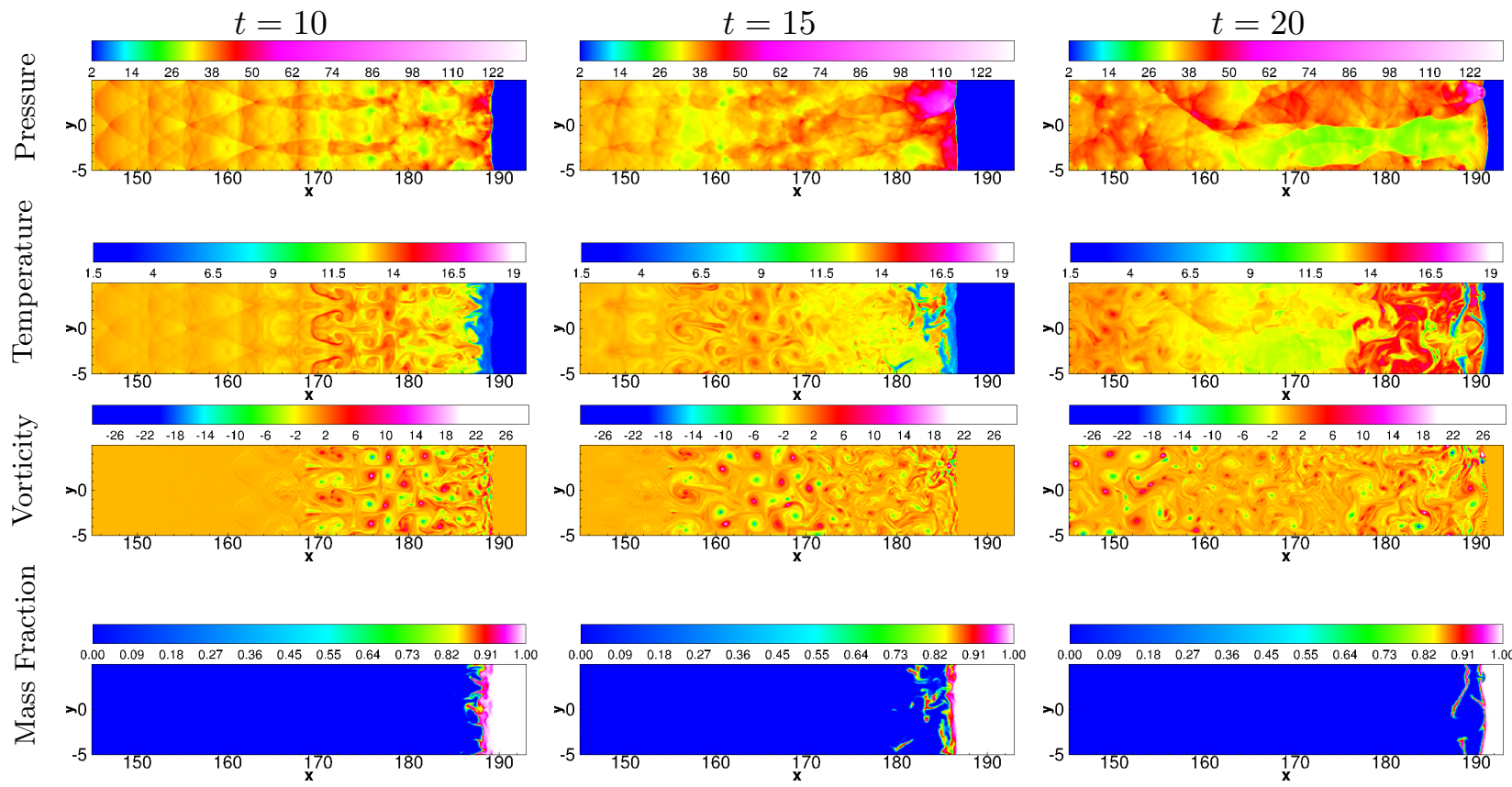

Figure 11: $\left(H_{50}, E_{50}, f_{1.2}\right)$ : (Color online) Contours of the pressure, temperature, vorticity and mass fraction at times $t=10,15,20$ with grid resolution $\delta_{20}$. The parameters used for the perturbation waves are $(k=2, A=0.2)$.

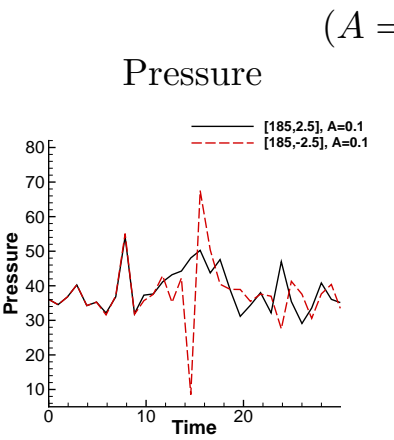

$$
\text { ( } A=0.1)
$$
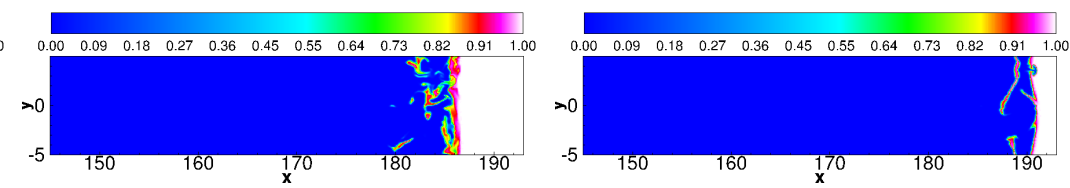

Figure 12: $\left(H_{50}, E_{50}, f_{1.2}\right)$ : (Color online) Pressures and velocities with the perturbation waves with parameters $(k=2, A=0.1),(k=2, A=0.2)$. The data are recorded at location $(185,2.5)$ and $(185,-2.5)$. 
The pressure, temperature, vorticity and mass fraction of the flow computed by WENO-Z scheme with grid resolution $\delta_{20}$ and $(k=3, A=0.1)$ are shown in the Fig. 13. One can see that the number of detonation cells depends on the wave number of the perturbation sinusoidal waves.
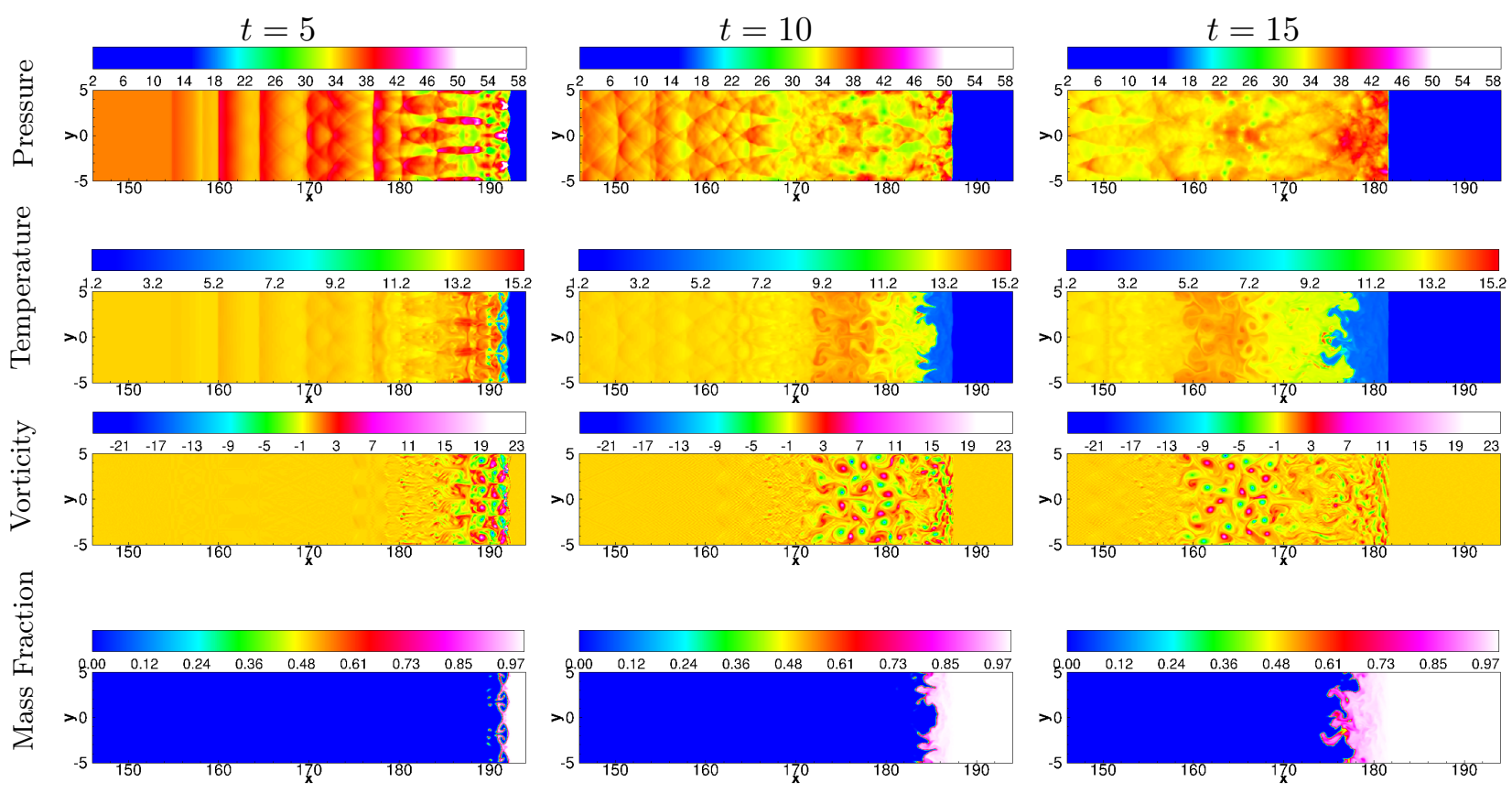

Figure 13: $\left(H_{50}, E_{50}, f_{1.2}\right)$ : (Color online) Contours of the pressure, temperature, vorticity and mass fraction at times $t=5,10,15$ with grid resolution $\delta_{20}$. The parameters of the perturbation waves are $(k=3, A=0.1)$.

\section{Conclusion and Future Work}

In this work, we studied the performance of the improved fifth order characteristic based weighted essentially non-oscillatory (WENO-Z) finite difference scheme for two dimensional detonation wave simulations. We presented some preliminary numerical studies of three examples with small heat release and low activation energy, large heat release and low activation energy, large heat release and large activation energy, respectively. The governing equations are a system of nonlinear hyperbolic conservation laws with a species production source term based on a simple irreversible chemical reaction. The heat generated by the chemical reaction is added to the total energy of the system, which drives the detonation front and vice versa. The behavior of the combustion front and the reaction zone behind the front depends exponentially on the local mass fraction and the temperature around the combustion front. The fifth order WENO-Z scheme with different grid resolutions and 
third order TVD Runge-Kutta scheme were employed to solve the nonlinear hyperbolic system in order to capture sharp detonation fronts and to resolve small scales structures that appear in the highly complex solution of flow. The computer program is written based on the FORTRAN 95 subroutines contained in the high performance software library WENOpack [37].

To reduce the computational cost and to avoid wave reflection from the artificial computational boundary of a truncated physical domain, we derive an efficient and easily implemented one dimensional Perfectly Matched Layer (PML) absorbing boundary condition (ABC) for the two dimensional unsteady reactive Euler equation when one of the direction of domain is periodical and inflow/outflow in the other direction. Numerical comparisons among characteristic, free stream, extrapolation and PML boundary conditions are conducted for the detonation wave simulations. It showed that PML boundary condition is the best choice to absorb the outgoing waves while minimizing the wave reflection from the artificial computational boundary.

Numerical schemes for solving the system of hyperbolic conversation laws which uses a singe mode sinusoidal perturbed ZND analytical solution as initial conditions are presented. We showed that the larger amplitude of perturbation wave generated more smaller scale structures of detonation wave due to more energy is released when the shock fronts came across the strong perturbation wave. The number of cell structures depends on the wave number of sinusoidal perturbation. Regular rectangular combustion cell, pockets of unburned gas and bubbles and spikes are generated and generally well resolved in the simulations. The present results from the simulations agree well with the published theoretical and numerical results in the literature.

In our future work in this area, a multi-modes perturbation and/or random perturbation within the reaction zone will be considered in order to study the complex detonation behavior. We will examine quantitative behavior of the system via the mixing profile, statistics and spectra of perturbation energy fields (for example, see [38]) in our future work.

\section{Acknowledgments}

The authors would like to acknowledge the funding support of this research by the RGC grant HKBU-200910 from Hong Kong University Grant Council. The first author gratefully acknowledges the invitation of Prof. Don, Wai-Sun and also extends his gratitude to the Department of Mathematics at Hong Kong Baptist University for hosting his visit. He also acknowledges Prof. Cheng Wang for many useful discussions throughout the research and the funding support of this research by the Fundamental Research Funds for the Central Universities (1501-841111012). 


\section{$\underline{\text { References }}$}

[1] D. Balsara and C. W. Shu, Monotonicity preserving weighted essentially non-oscillatory schemes with increasingly high order of accuracy, J. Comput. Phys. 160 (2000), pp. 405-452.

[2] A. Harten, High resolution schemes for hyperbolic conservation laws, J. Comput. Phys. 49 (1983), pp. 357-393.

[3] G. S. Jiang and C. W. Shu, Efficient Implementation of Weighted ENO Schemes, J. Comput. Phys. 126 (1996), pp. 202-228.

[4] A. K. Henrick, T. D. Aslam and J. M. Powers, Mapped weighted essentially non-oscillatory schemes: Achieving optimal order near critical points, J. Comput. Phys. 207 (2005), pp. 542 567.

[5] R. Borges, M. Carmona, B. Costa and W. S. Don, An improved weighted essentially nonoscillatory scheme for hyperbolic conservation laws, J. Comput. Phys. 227 (2008), pp. 31013211.

[6] Y. B. Zeldovich, On the theory of the propagation of detonation in gaseous system, Zh. Eksp. Teor. Fiz. 10 (1940), pp. 542-568.

[7] J. V. Neumann, Theory of Detonation Waves, John von Neumann, Collected Works, Macmillan, New York, 6 (1942).

[8] W. Doering, On detonation processes in gases, Ann. Phys. 43 (1943), pp. 421-436.

[9] J. J. Erpenbeck, Stability of steady-state equilibrium detonations, Phys. Fluids 5 (1962), pp. 604-614.

[10] A. K. Oppenheim and R. I. Soloukhin, Experiments in gasdynamics of explosions, Ann. Rev. Fluid Mech. 5 (1973), pp. 31-58.

[11] W. Fickett and W. C. Davis, Detonation, University of California Press, Berkeley (1979).

[12] J. H. S. Lee and I. O. Moen, The mechanism of transition from deflagration to detonation in vapor cloud explosion, Progr. Energy Combust. Sci. 6 (1980), pp. 359-389.

[13] F. Sharpe and S. A. E. G. Sharpe, Two-dimensional numerical simulations of idealized detonations, Proc. R. Soc. Lond. A 456 (2000), pp. 2081-2100.

[14] B. N. Azarenok and T. Tang, Second-order Godunov-type scheme for reactive flow calculations on moving meshes, J. Comput. Phys. 206 (2005), pp. 48-80.

[15] Z. C. Zhang, S. T. Yu, H. He and S. C. Chang, Direct calculation of two- and three- dimensional detonations by an extended CE/SE method, AIAA Paper 2001-0476 (2001). 
[16] M. V. Papalexandris, A. Leonard and P. E. Dimotakis, Unsplit Algorithms for Multidimensional System of Hyperbolic Conservation Laws with Source Terms, Comput. Math. Appl. 44 (2002), pp. 25-49.

[17] X. He and A. R. Karagozian Numerical Simulation of Pulse Detonation Engine Phenomena, J. Sci. Comput. 19 (2003), pp. 201-224.

[18] C. Wang, J. Ning and J. Lei, Numerical Study on Propagation of Explosion Wave in H2-O2 Mixtures, Adv. Nat. Comput. 4222 (2006), pp. 816-819.

[19] Z. Gao, Z. Li and W. S. Don, High Order Weighted Essentially Non-Oscillation Schemes for One-Dimensional Detonation Wave Simulations, J. Comput. Math., (http://www.math.hkbu.edu.hk/ICM/researchreports 11-02)

[20] A. Bourlioux, A. J. Majda and V. Roytburd, Theoretical and Numerical Structure for Unstable One-Dimensional Detonations, SIAM J. Appl. Math. 51 (1991), pp. 303-343.

[21] M. V. Papalexandris, A. Leonard and P. E. Dimotakis, Unsplit schemes for hyperbolic conservation laws with source terms in one space detonation, J. Comput. Phys. 134 (1997), pp. $31-61$.

[22] J. J. Quirk, Godunov-type schemes applied to detonation flows, ICASE Report 93-15 (1993).

[23] J. P. Berenger, A Perfectly Matched Layer for the Absorption of Electromagnetic waves, J. Comput. Phys. 114 (1994), pp. 185-200.

[24] J. S. Hesthaven, On the analysis and construction of perfectly matched layers for the linearized Euler equations, J. Comput. Phys. 142 (1998), pp. 129-147.

[25] T. Hagstrom, A new construction of perfectly matched layers for hyperbolic systems with applications to the linearized Euler equations, in: G. Cohen, E. Heikkola, P. Joly, P. Neittaanmäki (Eds.), Mathematical and Numerical Aspects of Wave Propagation Phenomena, SpringerVerlag, Berlin (2003), pp. 125-129.

[26] F. Q. Hu, A perfectly matched layer absorbing boundary condition for linearized Euler equations with a non-uniform mean-flow, J. Comput. Phys. 208 (2005), pp. 469-492.

[27] F. Q. Hu, On the construction of PML absorbing boundary condition for the non-linear Euler equations, AIAA Paper 2006-0798 (2006).

[28] M. E. Brachet, M. Meneguzzi and P. L. Sulem, Small-Scale Dynamics of High-ReynoldsNumber Two-Dimensional Turbulence, Phys. Rev. Lett. 57 (1986), pp. 683-686.

[29] M. Castro, B. Costa and W. S. Don, High order weighted essentially non-oscillatory WENO-Z schemes for hyperbolic conservation laws, J. Comput. Phys. 230 (2011), pp. 1766-1792. 
[30] H. S. Dou, H. M. Tsai, B. C. Khoo and J. Qiu, Simulations of Detonation Wave Propagation in Rectangular Ducts Using a Three-Dimensional WENO Scheme, Combust. Flame, 154 (2008), pp. 644-659.

[31] F. Q. Hu On Perfectly Matched Layer as an absorbing boundary condition, AIAA paper 961664 (1996).

[32] A. Bourlioux and A. J. Majda, Theoretical and Numerical Structure for Unstable TwoDimensional Detonations, Combust. Flame 90 (1992), pp. 211-229.

[33] E. F. Toro, Riemann solvers and numerical methods for fluid dynamics, A practical introduction, Springer, 1999.

[34] J. J. Erpenbeck, Stability of idealized one-reaction detonations, Phys. Fluids 9 (1964), pp. 684-696.

[35] H. I. Lee and D. S. Stewart, Calculation of Linear Detonation Instability, One-Dimensional Instability of Plane Detonation, J. Fluid Mech. 216 (1990), pp. 103-132.

[36] H. S. Dou, H. M. Tsai, B. C. Khoo and J. Qiu, Simulations of detonation wave propagation in rectangular ducts using a three-dimensional WENO scheme, Combust. Flame 154 (2008), pp. 644-659.

[37] W. S. Don, WENOpack, http://www.math.hkbu.edu.hk/ wsdon/.

[38] M. Latini, O. Schilling and W. S. Don, Effects of order of WENO flux reconstruction and spatial resolution on reshocked two-dimensional Richtmyer-Meshkov instability, J. Comput. Phys. 221 (2007), pp. 805-836. 